\title{
From polarized gravitational waves to analytically solvable electromagnetic beams
}

\author{
K. Andrzejewski ${ }^{*}$ and S. Prencel \\ Department of Computer Science, Faculty of Physics and Applied Informatics, University of Lodz, \\ Pomorska 149/153, 90-236, Lodz, Poland
}

(Received 29 January 2019; published 8 August 2019)

\begin{abstract}
Using the correspondence between solutions of gravitational and gauge theories (the so-called classical double copy conjecture) some electromagnetic fields with vortices are constructed, for which the Lorentz force equations are analytically solvable. The starting point is a certain class of plane gravitational waves exhibiting the conformal symmetry. The notion of the Niederer transformation, crucial for the solvability, is analyzed in the case of the Lorentz force equation on the curved spacetimes as well as its derivation by means of integrals of motion (associated with conformal generators preserving these vortices) is presented. Furthermore, some models discussed recently in the context of the intense laser beams are constructed from their gravitational counterparts, with the special emphasis put on the focusing property, and new solvable examples are presented.
\end{abstract}

DOI: 10.1103/PhysRevD.100.045006

\section{INTRODUCTION}

Gravitational waves have been intensively studied since the invention of general relativity. Recently they have gained a new interest, both due to their direct observations $[1,2]$ and because of new theoretical ideas such as the memory effect and soft graviton theorems ([3-10] and references therein). The circularly polarized ones seem to be particularly interesting as they may arise as the effect of coalescing black holes and neutron star merger or can be observed from the astrometric data [2,11]. The linearly polarized waves are, in turn, distinguished by their relative simplicity. Far from the source in the neighborhood of the detector one can approximate gravitational waves by the exact plane ones (assuming that the backreaction of the detector is negligible). Finally, the so-called impulsive gravitational waves seem to be of some importance [12-17].

On the other hand, it turns out that in the case of the exact gravitational waves mentioned above there are three special classes; they are defined by the maximal (in the nonflat case), seven-dimensional, conformal symmetry [18-20], and they are the only ones exhibiting the maximal conformal symmetry among all nontrivial vacuum solutions to the Einstein equations. One of these

\footnotetext{
*Corresponding author. k-andrzejewski@uni.lodz.pl

Published by the American Physical Society under the terms of the Creative Commons Attribution 4.0 International license. Further distribution of this work must maintain attribution to the author(s) and the published article's title, journal citation, and DOI. Funded by SCOAP ${ }^{3}$.
}

classes consists of a metric family describing linearly or circularly polarized gravitational pulses. This exceptional family admits the proper conformal transformations; moreover, it can be used to model impulsive gravitational waves with the Dirac delta profile. However, the most interesting property of this family is that the geodesic equations can be explicitly solved. In consequence, some gravitational phenomena (such as singularities, focusing, classical cross section, and the velocity memory effect) can be analyzed analytically, also in the Dirac delta limit $[17,21]$. Such a situation is strictly related to the existence of the so-called Niederer transformation [21-23] (see also Sec. II for some details).

In this work, based on the above-mentioned polarized gravitational pulses, we study the interaction of the electromagnetic field with a charged particle. The bridge between the gravitational waves and electromagnetic fields is provided by the idea of the classical double copy (a part of the color-kinematic duality; see [24-32] and references therein). At the quantum level (analyzed as the tree and few loops levels) this conjecture concerns the problem of how scattering amplitudes in gravity can be obtained from those in the gauge theory by replacing the color structure with the kinematical one. At the classical level, it consists of the mapping of the solutions of the Einstein equations into the solutions of the Yang-Mills equations; for example a KerrSchild-type relation of the double copy [24] where the linear structure of the Einstein equations for the Kerr-Schild metrics corresponds to Abelian gauge fields. One of the main examples of such a correspondence is provided by the plane gravitational waves; they correspond to the electromagnetic potentials which yield certain, nonplane and 
vacuum, electromagnetic fields. As we indicated above there is an exceptional family of the plane gravitational waves for which the geodesic equations can be analytically analyzed. In view of the double copy conjecture the following question arises: does the same situation hold for the motion of a charged particle in the electromagnetic fields corresponding to this class? The positive answer to this question is obtained in Sec. III. In consequence, by using the idea of double copy we obtain electromagnetic fields for which (as for its gravitational counterparts) the dynamics is explicitly integrable. Moreover, the fields obtained exhibit vortices and generalize the ones described in Ref. [33] (which seem to be of some importance for singular optics and trapping problems, i.e., the confinement mechanism of particles by electromagnetic or gravitational fields; see [34,35] for the electromagnetic case and [36-38] for its gravitational counterpart). It is also worthwhile to notice that for the electromagnetic fields under consideration the Dirac delta limit of the profile can easily be performed.

Since the solvability discussed above is strictly related to the notion of the Niederer transformation we discuss in Sec. IV, a geometric extension of the Niederer map to the case of the plane gravitational spacetimes endowed with some, crossed, electromagnetic fields. As a consequence we obtain some examples of Lorentz force equations in suitable gravitational backgrounds which are solvable in the transverse directions.

In Sec. V we slightly modify the Kerr-Schild ansatz to produce non-null electromagnetic fields. Such electromagnetic fields have been discussed in Refs. [39,40]; they capture some essential features of the transverse magnetic beam of laser light near the beam axis. Next, we apply this procedure to the linearly polarized gravitational metrics and obtain an explicitly solvable model described in Ref. [39]. Moreover, relying on a member of the family of circularly polarized gravitational pulses we construct a transversally solvable electromagnetic background. However, in contrast to the previous one it has zeros; this fact essentially modifies the focusing conditions important for intense or ultrashort laser pulses (when the paraxial approximation may no longer be valid; see e.g., [41,42]). In the general case, we give some criteria that were applied to the cases under consideration.

Moreover, in Sec. VI the role and meaning of integrals of motion are discussed with the special emphasis on new ones associated with conformal generators preserving vortices under discussion; it is shown that these integrals lead to the Ermakov-Lewis invariants [43-45] and consequently to the Niederer transformation. The results outlined above suggest a deeper connection (related to the conformal symmetry and integrability) between gravitational and gauge theories rooted in the double copy approach. Finally, in Sec. VII we give a summary with an outlook for further studies.

\section{PLANE GRAVITATIONAL WAVES AND CONFORMAL SYMMETRY}

In this section we recall some facts crucial for our further studies. They concern the role of the conformal symmetry in the problem of analytical solutions of the geodesic equations. Namely, let us consider a subclass of the $p p$ waves, the so-called generalized plane gravitational waves, of the following form ${ }^{1}$ :

$$
g=\mathbf{x} \cdot H(u) \mathbf{x} d u^{2}+2 d u d v+d \mathbf{x} \cdot d \mathbf{x},
$$

where $H$ is assumed, without loss of generality, to be a symmetric matrix. In general, they are solutions to Einstein's field equations in which the only source of gravity is some kind of radiation; i.e., the source is a null fluid. The weak energy condition implies $\operatorname{tr}(H) \leq 0$ and the scalar curvature vanishes. If $\operatorname{tr}(H)=0$, then $g$ satisfies the vacuum Einstein equations and, consequently, describes a plane gravitational wave (exact gravitational wave). The geodesic equations for the metric (2.1) reduce to the following ones:

$$
\begin{gathered}
\ddot{\mathbf{x}}=H \mathbf{x}, \\
\ddot{v}=-\frac{1}{2} \mathbf{x} \cdot \dot{H} \mathbf{x}-2 \mathbf{x} \cdot H \dot{\mathbf{x}},
\end{gathered}
$$

where the dot refers to the derivative with respect to $u$. Furthermore, Eq. (2.3) can be directly integrated yielding

$$
v(u)=-\frac{1}{2} \mathbf{x} \cdot \dot{\mathbf{x}}+C_{1} u+C_{2},
$$

where $\mathbf{x}$ is a solution to (2.2). Thus for the (generalized) plane gravitational waves the solution to geodesic equations is obtained by solving the set of Eqs. (2.2). Moreover, the latter are particularly interesting in the case of the exact gravitational waves since they coincide with the deviation equations and enter the transformation rules to the so-called Baldwin-Jeffery-Rosen coordinates [5,6]. Although this set of equations cannot, in general, be explicitly solved, there are some special cases when the solution is accessible (see e.g., the classical paper [46]). Another, more geometric approach to this problem is related to the symmetry of the metric; the most interesting cases are the ones exhibiting the maximal symmetry. Let us start with the isometry groups. It is well known that the generic dimension of the isometry group of plane gravitational waves is five. There exist two exceptional families admitting the sixdimensional isometry groups. The first one is defined by the matrix $H$ of the form

\footnotetext{
${ }^{1}$ We use the following conventions: signature $(-,+,+,+)$ and $u \equiv\left(x^{3}-x^{0}\right) / \sqrt{2}$; bold indices refer to two-dimensional vectors, and the Einstein convention is assumed.
} 


$$
H^{(0)}(u)=\left(\begin{array}{cc}
\cos (\kappa u) & \sin (\kappa u) \\
\sin (\kappa u) & -\cos (\kappa u)
\end{array}\right)
$$

it admits the explicit solutions to the geodesic equations (cf. [16,47] and references therein). The second family describes geodesically incomplete manifolds defined by $H \sim u^{-2}$; it is intensively used in the context of the Penrose limit $[48,49]$.

The situation becomes more interesting if we take into account the conformal symmetry. Let us recall that the maximal dimension of the conformal group of the nonconformally flat metric is seven [50]. Note that the metric (2.1) admits a homothetic vector field. Therefore, the two above-mentioned families exhibit the seven-dimensional conformal symmetry (six isometries and homothety). It appears that there exists only one subclass of the plane gravitational waves with the seven-dimensional conformal group consisting of five-dimensional isometry, homothety, and nonhomothetic conformal transformations (cf. [18-20] and [51-53]). This special subclass consists of two families describing linearly and circularly polarized plane gravitational waves. In the following, we concentrate on them (actually, on the geodesically complete cases as the most interesting ones). The first family, linearly polarized, is defined by the metric $g^{(1)}$ with the profile

$$
H^{(1)}(u)=\frac{a}{\left(u^{2}+\epsilon^{2}\right)^{2}}\left(\begin{array}{cc}
1 & 0 \\
0 & -1
\end{array}\right),
$$

where $\epsilon>0$ and $a$ is an arbitrary number (excluding the trivial Minkowski case and changing $x^{1}$ and $x^{2}$ one can assume $a>0$ ). Moreover, let us note that taking $a \sim \epsilon^{3}$ one obtains the impulsive gravitational wave with the Dirac delta profile (as $\epsilon$ tends to zero).

The second family $g^{(2)}$ is an example of the circularly polarized plane gravitational waves. It is defined by the following profile:

$$
H^{(2)}(u)=\frac{a}{\left(u^{2}+\epsilon^{2}\right)^{2}}\left(\begin{array}{cc}
\cos (\phi(u)) & \sin (\phi(u)) \\
\sin (\phi(u)) & -\cos (\phi(u))
\end{array}\right),
$$

where

$$
\phi(u)=\frac{2 \gamma}{\epsilon} \tan ^{-1}(u / \epsilon),
$$

$\epsilon, \gamma>0$ and $a$ can be chosen as above [for $\gamma=0$, Eq. (2.7) reduces to the previous case; however, for physical and mathematical reasons we shall consider linear and circular polarizations separately].

Some properties of the gravitational waves $g^{(1,2)}$ were discussed in $[17,21]$. Among others it was noticed that the geodesic equations can be explicitly solved; furthermore, it was shown that this fact can be simply explained in terms of the so-called Niederer transformation (see also Sec. IV). In the case of $g^{(1)}$ the transversal part of the geodesics reads

$$
x^{i}(u)=C_{1}^{i} \sqrt{u^{2}+\epsilon^{2}} \sin \left(\Lambda_{i} \tan ^{-1}(u / \epsilon)+C_{2}^{i}\right),
$$

where

$$
\Lambda_{i}=\sqrt{1+(-1)^{i} \frac{a}{\epsilon^{2}}}, \quad i=1,2 .
$$

Moreover, the initial conditions

$$
\dot{\mathbf{x}}(-\infty)=0, \quad \mathbf{x}(-\infty)=\mathbf{x}_{\text {in }}
$$

lead to the observation that only the second component $x^{2}(u)$ exhibits focusing. In the case of $g^{(2)}$, the solutions are of the form

$$
\mathbf{x}(u)=\frac{\epsilon R(\tilde{u}) \mathbf{y}(\tilde{u})}{\cos (\tilde{u})}, \quad \tilde{u}=\tan ^{-1}(u / \epsilon),
$$

where

$$
R(\tilde{u})=\left(\begin{array}{cc}
\cos (\omega \tilde{u}) & -\sin (\omega \tilde{u}) \\
\sin (\omega \tilde{u}) & \cos (\omega \tilde{u})
\end{array}\right), \quad \omega=\frac{\gamma}{\epsilon},
$$

and $y$ 's are solutions to the following set of differential equations with constant coefficients:

$$
\begin{aligned}
& \left(y^{2}\right)^{\prime \prime}+2 \omega\left(y^{1}\right)^{\prime}+\Omega_{-} y^{2}=0, \\
& \left(y^{1}\right)^{\prime \prime}-2 \omega\left(y^{2}\right)^{\prime}+\Omega_{+} y^{1}=0,
\end{aligned}
$$

with

$$
\Omega_{ \pm}=1-\omega^{2} \mp \Omega, \quad \Omega=\frac{a}{\epsilon^{2}}
$$

here primes refer to the derivatives with respect to $\tilde{u}$. Although the general solution of Eqs. (2.14) is well known, in our case it is a linear combination of trigonometric functions only or both hyperbolic (or linear) and trigonometric ones depending on the values of parameters appearing in (2.14), the form of the coefficients can be quite complicated (for $\gamma=\epsilon$ they are presented in [21]). In the general case the transformation to the normal coordinates seems more useful (see also [54,55]). The above results form a setup for our further considerations.

\section{EXACTLY SOLVABLE ELECTROMAGNETIC VORTICES}

In the previous section we pointed out that for the plane gravitational pulses exhibiting the maximal conformal symmetry the geodesic equations can be analytically solved. 
Here, we showed that a similar situation holds for the electromagnetic fields constructed by means of the double copy conjecture; i.e., the existence of explicit solutions to the geodesic equations coincide with the existence of the explicit solutions to the Lorentz force equations for the corresponding electromagnetic backgrounds. To this end let us consider, in the Minkowski spacetime and light-cone coordinates, the following electromagnetic one-form:

$$
\mathcal{A}=-\mathbf{x} \cdot A(u) \mathbf{x} d u .
$$

Such a potential yields the (crossed) electromagnetic fields

$$
\vec{E}=\left(f_{1}, f_{2}, 0\right), \quad \vec{B}=\left(-f_{2}, f_{1}, 0\right),
$$

where

$$
\mathbf{f}=\left(f_{1}, f_{2}\right)=\left(\sqrt{2} A_{1 i} x^{i}, \sqrt{2} A_{2 i} x^{i}\right) .
$$

The fields $\vec{E}$ and $\vec{B}$ satisfy Maxwell's equations with the following null current:

$$
j^{\mu}=\sqrt{2} \operatorname{tr}(A)(1,0,0,1), \quad j^{\mu} j_{\mu}=0
$$

(cf. $[56,57])$.

Furthermore, the following conditions:

$$
\vec{E}^{2}-\vec{B}^{2}=0, \quad \vec{E} \cdot \vec{B}=0,
$$

hold and imply that the electromagnetic field is the pure radiation (the energy density and the Poynting vector form a null four-vector). When $\operatorname{tr}(A) \neq 0$, e.g., $A$ is proportional to the identity, then $j^{\mu}$ can be interpreted as a pulse of charges of one sign moving with the speed of light along the $z$-axis, especially when a suitable regularization is performed ( $j^{\mu}$ is zero outside a transversal region); unfortunately, even then the total energy of fields is infinite. The situation changes if a dipole model of the particle is taken under consideration; for more details see the original paper [58].

The case $\operatorname{tr}(A)=0$ corresponds to the electromagnetic field satisfying the vacuum Maxwell equations; however, such fields are not, in general, electromagnetic plane waves. Moreover, for arbitrary vacuum solutions the conditions (3.5) (equivalently the vanishing of the square of the Riemann-Silberstein vector) can be used to describe the vortex of the electromagnetic field [59]. However, in the null fluid case, i.e., when (3.5) vanishes identically, the notion of the vortex can be simplified; it is then related to the condition that all components of the electromagnetic field vanish (see [60]). In this approach the electromagnetic field (3.2) carries a straight vortex line along the $z$-axis. This is interesting due to the fact that the electromagnetic vortices (vortex lines) gained some attention not only in the context of singular optics but also in the confinement mechanisms of particles by electromagnetic fields or even knot theory; see [33-35] and references therein.

Now let us note that for the potential (3.1) the Lorentz force equations

$$
m \frac{d^{2} x^{\mu}}{d \tau^{2}}=e F_{\nu}^{\mu} \frac{d x^{\nu}}{d \tau}
$$

give

$$
\begin{gathered}
m \frac{d^{2} \mathbf{x}}{d \tau^{2}}=e \mathbf{f} \frac{d}{d \tau}\left(x^{0}-x^{3}\right), \\
\frac{1}{\sqrt{2}}\left(\frac{d x^{0}}{d \tau}-\frac{d x^{3}}{d \tau}\right)=-\frac{d u}{d \tau}=\frac{-p_{v}}{m}>0
\end{gathered}
$$

( $p_{v}<0$ is the light-cone momentum of the particle); and consequently Eqs. (3.6) can be expressed in terms of the $u$ coordinate as follows:

$$
\begin{aligned}
\ddot{\mathbf{x}} & =\frac{2 e}{-p_{v}} A \mathbf{x}, \\
\ddot{v} & =\frac{2 e}{p_{v}} \mathbf{x} \cdot A \dot{\mathbf{x}}
\end{aligned}
$$

(see also [56,57]). Thus, up to the constant $\frac{2 e}{-p_{v}}$, the transverse part of the equation of motion (3.9) has the same form (in contrast to the longitudinal direction; see the discussion below) as for the plane gravitational waves [cf. Eqs. (2.2)].

The above properties of the vacuum electromagnetic field given by the potential (3.1) can be considered as a manifestation of the idea of double copy which at the quantum level concerns the problem of how scattering amplitudes in gravity can be obtained from those in the gauge theory by replacing the color structure with the kinematical one; for more details we refer to some surveys $[29,30]$ because our further considerations will focus on its classical counterparts which have been proposed (see e.g., [24-28]) to better understand this conjecture (for example whether the copy is a genuinely nonperturbative property of both theories). In this approach we look directly at solutions of the classical field equations in gauge and gravity theories and match these up according to a double copy prescription. Such a matching has been observed for the metrics in the so-called Kerr-Schild coordinates [24], more precisely, for the metrics which can be written in the following form:

$$
g_{\mu \nu}=\bar{g}_{\mu \nu}+\lambda k_{\mu} k_{\nu},
$$


where $\lambda$ is a scalar function and $k^{\mu}$ is a null vector with respect to both metrics. It turns out that under assumption $k^{\mu} \bar{D}_{\mu} k_{\nu}=0$ the Ricci tensor has the remarkable property that it is linear in $\lambda$. This property suggests that the corresponding gauge field should take the simplest form (even an Abelian one). For the Minkowski background $\bar{g}=\eta$ the following (in general non-Abelian ansatz) was proposed:

$$
A_{\mu}^{a}=c^{a} \lambda k_{\mu}
$$

where $c^{a}$ is an arbitrary constant color vector. In this case under some additional assumptions concerning $\lambda$ and $k_{\mu}$ (e.g., stationary) vacuum solutions are invariant under this correspondence. Although it is not known how to fully extend the above case, several attempts have been made (including AdS background, Taub-Nut, or Kundt spacetimes; see $[25,26,31,32])$. One of the most natural extensions is based on the metric of the following form:

$$
g_{\mu \nu}=\bar{g}_{\mu \nu}+\lambda_{1} k_{\mu} k_{\nu}+\lambda_{2} l_{\mu} l_{\nu}
$$

where $l^{\mu}$ is also a null vector and orthogonal, with respect to $g$ and $\bar{g}$, to $k^{\mu}$. For such a metric the corresponding gauge potential (in $\bar{g}$ spacetime) reads

$$
A_{\mu}^{a}=c^{a}\left(\lambda_{1} k_{\mu}+\lambda_{2} l_{\mu}\right)
$$

One of the main examples of the correspondence outlined above is provided by the plane gravitational waves and nonplane electromagnetic fields. Namely, taking $\bar{g}=\eta$ and the vector $k^{\mu}=(1,0,0,1) / \sqrt{2}$, the generalized plane wave

(2.1) takes the form (3.11) with $\lambda=\lambda(u, \vec{x})=\mathbf{x} \cdot H(u) \mathbf{x}$. According to the classical double copy conjecture the corresponding electromagnetic potential (3.12) coincides with (3.1) if we identify the matrices $A=H$ (in the simplest Abelian, case). In Sec. IV we use this correspondence taking a plane wave spacetime (2.1) as a fixed background $\bar{g}$; then the additional term in (3.11) can be interpreted as the potential in curved spacetime $\bar{g}$ [cf. (3.13) and see also a more detailed discussion in Refs. [25,26] ].

The above outlined reasoning has been used in Ref. [36] to study an electromagnetic vortex proposed in [33] which can act as a beam guide for charged particles; moreover, it is analytically solvable (see also [56]) as well as provides an approximation to more realistic beams [61]. Such a vortex corresponds to the plane gravitational wave, defined by the profile (2.5), which is also analytically solvable. Guided by the above idea of classical double copy in what follows, we show that the vortex mentioned possesses solvable extensions based on the gravitational waves related to the proper conformal symmetry discussed in the previous section.

To this end let us consider the electromagnetic potentials related to the plane gravitational waves $H^{(1,2)}$. Then, by virtue of (2.6), (2.7), and (3.1)-(3.3), one obtains the following electromagnetic fields in the Minkowski spacetime:

$$
\begin{aligned}
& \vec{E}^{(1)}(x)=\frac{\sqrt{2} a}{\left(u^{2}+\epsilon^{2}\right)^{2}}\left(x^{1},-x^{2}, 0\right), \\
& \vec{B}^{(1)}(x)=\frac{\sqrt{2} a}{\left(u^{2}+\epsilon^{2}\right)^{2}}\left(x^{2}, x^{1}, 0\right) ;
\end{aligned}
$$

$$
\begin{aligned}
& \vec{E}^{(2)}(x)=\frac{\sqrt{2} a}{\left(u^{2}+\epsilon^{2}\right)^{2}}\left(x^{1} \cos (\phi(u))+x^{2} \sin (\phi(u)), x^{1} \sin (\phi(u))-x^{2} \cos (\phi(u)), 0\right), \\
& \vec{B}^{(2)}(x)=\frac{\sqrt{2} a}{\left(u^{2}+\epsilon^{2}\right)^{2}}\left(-x^{1} \sin (\phi(u))+x^{2} \cos (\phi(u)), x^{1} \cos (\phi(u))+x^{2} \sin (\phi(u)), 0\right),
\end{aligned}
$$

where $\phi$ is given by (2.8) and $u=\left(x^{3}-x^{0}\right) / \sqrt{2}$. As it has been indicated in Sec. II, the geodesics equations for $g^{(1,2)}$ are analytically solvable; in consequence one immediately [after replacing $a \mapsto \frac{2 e}{-p_{v}} a$; see (2.2) and (3.9)] obtains the solution to the transverse Lorentz force equations with $\vec{E}^{(1,2)}$ and $\vec{B}^{(1,2)}$ [cf. Eqs. (2.9) and (2.12)-(2.15)]. Finally, taking an appropriate limit (cf. $[17,21]$ ) one gets the case of an electromagnetic field with the profile proportional to the Dirac delta function $\delta(u)$.

Now, let us consider the longitudinal direction. In contrast to the gravitational case, Eq. (3.10) can be directly integrated only once,

$$
\dot{v}=-\frac{1}{2} \dot{\mathbf{x}} \cdot \dot{\mathbf{x}}+D_{1}
$$

However, in what follows we show that for the field given by (3.15) and (3.16) it is also possible to find explicitly the $v$ coordinate. Indeed, integrating (3.17) by substitution $\tilde{u}=\tan ^{-1}(u / \epsilon)$ one obtains

$$
v(u)=D_{1} u+D_{2}-\frac{1}{2 \epsilon} \int \mathbf{x}^{\prime}(\tilde{u}) \cdot \mathbf{x}^{\prime}(\tilde{u}) \cos ^{2}(\tilde{u}) d \tilde{u} .
$$


Now, for the fields $\vec{E}^{(1)}, \vec{B}^{(1)}$ using (2.9) one gets, after some computations, the following final form of the longitudinal coordinate:

$$
v(u)=-\frac{\epsilon}{2} \sum_{i}\left(C_{1}^{i}\right)^{2}\left(\frac{1}{2}\left(b_{i}^{2}-1\right) \tilde{u}+\sin ^{2}\left(b_{i} \tilde{u}+C_{2}^{i}\right) \tan (\tilde{u})+\frac{1+b_{i}^{2}}{4 b_{i}} \sin \left(2\left(b_{i} \tilde{u}+C_{2}^{i}\right)\right)\right)+D_{2}+D_{1} u,
$$

where $\tilde{u}=\tan ^{-1}(u / \epsilon)$ and $b_{i}=\sqrt{1-(-1)^{i} \frac{2 e a}{\epsilon^{2} p_{v}}}$.

The second case is slightly more involved. First, we express the integral in (3.18) in terms of $y$ 's satisfying Eqs. (2.14) (with $\Omega=\frac{2 e a}{-p_{v} \epsilon^{2}}$ ), and, subsequently, we extract a total time derivative term; in consequence we arrive at the following equalities:

$$
\begin{aligned}
- & \frac{1}{\epsilon^{2}} \int \mathbf{x}^{\prime}(\tilde{u}) \cdot \mathbf{x}^{\prime}(\tilde{u}) \cos ^{2}(\tilde{u}) d \tilde{u} \\
& =\int\left[\left(\left(y^{1}\right)^{\prime}-\omega y^{2}\right)^{2}+\left(\left(y^{2}\right)^{\prime}+\omega y^{1}\right)^{2}+2 \tan (\tilde{u})\left(\left(y^{1}\right)^{\prime} y^{1}+\left(y^{2}\right)^{\prime} y^{2}\right)+\tan ^{2}(\tilde{u})\left(\left(y^{1}\right)^{2}+\left(y^{2}\right)^{2}\right)\right] d \tilde{u} \\
& \left.=\left(y^{2}\right)^{\prime} y^{2}+\left(y^{1}\right)^{\prime} y^{1}+\left(\left(y^{1}\right)^{2}+\left(y^{2}\right)^{2}\right) \tan (\tilde{u})+\Omega \int\left[\left(y^{2}\right)^{2}-\left(y^{1}\right)^{2}\right)\right] d \tilde{u} .
\end{aligned}
$$

Let us note that $y$ 's are combinations of the trigonometric or hyperbolic functions [cf. (2.14)]; thus the last integral in (3.20) is an elementary one and can be explicitly computed. Finally, substituting $\tilde{u}=\tan ^{-1}(u / \epsilon)$ in (3.18) one obtains the form of $v(u)$ which gives the analytical solvability of the Lorentz force equations in the electromagnetic backgrounds (3.15) and (3.16) (extending in this way some results obtained in Ref. [33] to a kind of electromagnetic pulses exhibiting vortices).

\section{NIEDERER'S MAP AND LORENTZ'S FORCE EQUATION}

It turns out that [21] the existence of analytical solutions to the geodesic equations for the gravitational waves $g^{(1,2)}$ can be simply explained in terms of the so-called Niederer transformation $[22,23]$. We shall show that the similar situation holds also when the electromagnetic fields (3.15) or (3.16) are switched on. To this end and to make the paper more self-contained let us recall some facts concerning the Niederer map and its geometric interpretation. The Niederer transformation, ${ }^{2}$

$$
\begin{aligned}
& u=\epsilon \tan (\tilde{u}), \\
& \mathbf{x}=\frac{\epsilon \tilde{\mathbf{x}}}{\cos (\tilde{u})},
\end{aligned}
$$

relates the free motion $\ddot{\mathbf{x}}=0$ (for our purpose we consider the two-dimensional case) on the whole real axis

\footnotetext{
${ }^{2}$ For further considerations we adopt $u$-notation. There exists a hyperbolic counterpart of Niederer's transformation leading to the repulsive case.
}

$(-\infty<u<\infty)$ to the half of the period motion $\left(-\frac{\pi}{2}<\tilde{u}<\frac{\pi}{2}\right)$ of the attractive harmonic motion $\tilde{\mathbf{x}}^{\prime \prime}=$ $-\tilde{\mathbf{x}}$, as the above dot and prime refer to the derivatives with respect to $u$ and $\tilde{u}$, respectively (this equivalence also continues to hold at the quantum level [22]). Of course, the above observation has a local character; however, it reflects a similarity between both systems and brings some useful information. Various local quantities can be directly related; this concerns even the global ones (for instance, Feynman propagators) if sufficient care is exercised (see e.g., $[22,62,63])$. In particular, the maximal symmetry groups of both systems are isomorphic and one obtains the explicit relation between symmetry generators as well as solutions of both systems [64].

On the other hand, Eqs. (2.2) describe, in fact, a linear oscillator (in general) with time-dependent frequencies; however, it turns out that in some cases the Niederer mapping can also be applied to relate them to a harmonic (or a simpler linear) oscillator [21]. Namely, under the Niederer transformation Eqs. (2.2) are transformed into the following ones:

$$
\tilde{\mathbf{x}}^{\prime \prime}=\tilde{H}(\tilde{u}) \tilde{\mathbf{x}}
$$

where

$$
\tilde{H}(\tilde{u})=\frac{\epsilon^{2} H(\epsilon \tan (\tilde{u}))}{\cos ^{4}(\tilde{u})}-I .
$$

In particular, if the matrix $H$ is of the form 


$$
H(u)=\frac{a}{\left(\epsilon^{2}+u^{2}\right)^{2}} G(u)
$$

where $G$ is a symmetric matrix, then, by virtue of Eq. (4.3), the equations of motion (4.2) read

$$
\tilde{\mathbf{x}}^{\prime \prime}=\tilde{H}(\tilde{u}) \tilde{\mathbf{x}}=\left(\frac{a}{\epsilon^{2}} G(\epsilon \tan (\tilde{u}))-I\right) \tilde{\mathbf{x}}
$$

For example, the (nonsingular) time-dependent linear oscillator defined by a constant matrix $G$ is mapped under (4.1) to a part of motion of the harmonic oscillator. In consequence the Niederer transformation can be useful to solve some geodesic equations for plane gravitational waves (or other problems where time dependent linear oscillators occur). Moreover, such properties of the Niederer transformation have a reflection in a geometric picture obtained by means of the Eisenhart-Duval lift [23,65] (see also [66,67] and references therein). Namely, extending the Niederer map by adding the following transformation rule:

$$
v=\epsilon \tilde{v}-\frac{\epsilon \tan (\tilde{u})}{2} \tilde{\mathbf{x}}^{2},
$$

one gets the identity

$$
\begin{aligned}
g & \equiv \mathbf{x} \cdot H(u) \mathbf{x} d u^{2}+2 d u d v+d \mathbf{x}^{2} \\
& =\frac{\epsilon^{2}}{\cos ^{2}(\tilde{u})}\left(\tilde{\mathbf{x}} \cdot \tilde{H}(\tilde{u}) \tilde{\mathbf{x}} d \tilde{u}^{2}+2 d \tilde{u} d \tilde{v}+d \tilde{\mathbf{x}}^{2}\right) \equiv \frac{\epsilon^{2}}{\cos ^{2}(\tilde{u})} \tilde{g},
\end{aligned}
$$

where $H$ and $\tilde{H}$ are connected by Eq. (4.3). For the particular case $H=0$, Eq. (4.7) reduces to the well-known relation between the Bargmann spacetimes corresponding to the free $(u, \mathbf{x}, v)$ and the half-oscillatory period $(\tilde{u}, \tilde{\mathbf{x}}, \tilde{v})$ motions in the Eisenhart-Duval lift language [23] (see also [68,69]).

In view of Eq. (4.7), by means of the Niederer transformation one can associate with the metric $g$ the new one $\tilde{g}$, conformally related to $g$, belonging to the same class (generalized plane gravitational waves). Of course, at most only one of the metrics $g$ and $\tilde{g}$ describes the vacuum solution; moreover, the geodesic equations for $g$ and $\tilde{g}$ are not equivalent (except those for null geodesics). However, from the reasoning underlying the Niederer transformation it follows that the transversal geodesic equations (2.2) for $g$ are mapped into the transversal geodesic equations (4.2) for the metric $\tilde{g}$, which may be more tractable than those for $g$. The nonequivalence of the geodesic equations for $g$ and $\tilde{g}$ is reduced to the nonequivalence of equations determining $v$ and $\tilde{v}$ [however, the latter can be easily solved; cf. Eq. (2.4)]. Such a situation holds, for instance, for the conformally distinguished metric families: $g^{(1,2)}$ and the one defined by $G \sim I$ (see [17,21]).
Now, we extend the geometric picture of the Niederer transformation outlined above to the case of both gravitational and electromagnetic backgrounds; as a consequence we obtain some examples of the Lorentz force equations in suitable gravitational backgrounds which are solvable in the transverse directions. Namely, let us analyze the action of Niederer's transformation on the spacetime described by the metric (2.1) (in particular, for $H=0$-the Minkowski one) endowed with the electromagnetic field given by the potential (3.1). First let us note that the condition $\operatorname{tr}(A)=0$ implies that, as in the Minkowski case, the electromagnetic field under consideration satisfies the vacuum Maxwell equations on the spacetime defined by a plane gravitational wave (this fact is valid even in the case of an arbitrary $p p$ wave spacetime). Furthermore, in this case the equations of motion of a charged test particle

$$
\frac{d^{2} x^{\alpha}}{d \tau^{2}}+\Gamma_{\nu \mu}^{\alpha} \frac{d x^{\nu}}{d \tau} \frac{d x^{\mu}}{d \tau}=\frac{q}{m} g^{\alpha \nu} F_{\nu \mu} \frac{d x^{\mu}}{d \tau}
$$

reduce to the following ones:

$$
\ddot{\mathbf{x}}=\left(H-\frac{2 e}{p_{v}} A\right) \mathbf{x},
$$

$$
\ddot{v}=-\frac{1}{2} \mathbf{x} \cdot \dot{H} \mathbf{x}-2 \mathbf{x} \cdot\left(H-\frac{e}{p_{v}} A\right) \dot{\mathbf{x}}
$$

[for the Minkowski spacetime they coincide with Eqs. (3.9) and (3.10)].

From the above we see that the transversal part of the Lorentz force equation is still decoupled and can be considered separately. Thus we can try to extend the geometric interpretation and applications of Niederer's transformation to electromagnetic fields in curved spacetimes. To this end let us note that under the Niederer transformation the transverse set of Eqs. (4.9) is mapped to the following ones:

$$
\tilde{\mathbf{x}}^{\prime \prime}=\left(\tilde{H}(\tilde{u})+\frac{2 e}{p_{v}} \tilde{A}(\tilde{u})\right) \tilde{\mathbf{x}},
$$

where $\tilde{H}$ is given by (4.3) while

$$
\tilde{A}(\tilde{u})=\frac{\epsilon^{2}}{\cos ^{4}(\tilde{u})} A(\epsilon \tan (\tilde{u})) .
$$

The solutions to Eqs. (4.11) corresponding to the purely gravitational case, i.e., $A=0$ and $g=g^{(1,2)}$, have been discussed in $[17,21]$ (see Sec. II), while for the Minkowski spacetime they have been endowed with the electromagnetic field given by (3.15) and (3.16) in Sec. III. However, by considering these gravitational and electromagnetic backgrounds altogether (i.e., $\vec{E}^{(i)}, \vec{B}^{(i)}$ on the spacetime $g^{(i)}$, for $i=1,2$, respectively) one finds that in 
these cases the transverse Lorentz force equations, given by (4.11), are also analytically solvable.

Next, by virtue of (4.7) the Niederer map transforms the metric $g$ conformally into the metric $\tilde{g}$ of the same type; i.e., we do not leave out the class of the generalized plane gravitational waves. For the electromagnetic potential (3.1) one obtains

$$
\mathcal{A}=-\mathbf{x} \cdot A(u) \mathbf{x} d u=\epsilon \tilde{\mathbf{x}} \cdot \tilde{A}(\tilde{u}) \tilde{\mathbf{x}} d \tilde{u},
$$

where $\tilde{A}$ is defined by (4.12). Thus the potential $\tilde{A}$, in the new coordinates, is also of the same type as $A$ and, consequently, yields also a crossed electromagnetic field. Moreover, the pair $(\tilde{g}, \tilde{A})$ is the one for which the transverse part of the Lorentz force equations is given by Eq. (4.11) (let us note that since $\tilde{g}$ is conformally related to $g$, the electromagnetic field arising from the potential $\tilde{A}$ is a vacuum solution to the Maxwell equation with respect to $\tilde{g}$, in contrast to the metric case where the vacuum solution $g$ is mapped to the nonvacuum null-fluid solution $\tilde{g}$ ). In this way we extend the notion of the Niederer transformation, originally established for the nonrelativistic dynamical systems, to the one including electromagnetic fields on curved spacetimes; namely with the pair $(g, A)$ we associate the new one $(\tilde{g}, \tilde{A})$ such that the transverse part of the Lorentz force equations for $(g, A)$ transform, by means of the Niederer map, into the one for $(\tilde{g}, \tilde{A})$ (moreover $g, \tilde{g}$ and $A, \tilde{A}$ belong to the same classes: the generalized plane gravitational waves and vacuum crossed electromagnetic fields, respectively).

\section{LIGHT-MATTER INTERACTION}

In this section we touch upon some problems of the interaction between optical beams (such as lasers) and charged particles. To this end let us recall that in the standard approach the optical beams (including laser beams or pulses) are described by the plane electromagnetic waves. Thus the dynamics of a charged particle in the presence of laser pulses reduces, at the classical level, to the solution of the Lorentz force equations and, at the quantum level, to the Dirac (Klein-Gordon) equation or, in general, QED process, in these fields. However, the real laser beam is localized (a finite beamwidth), and it has a high amplitude near the propagating direction and is less far away (transverse spatial variations); moreover, the beamwidth increases along the optical axis, and thus the wave fronts show a spherical nature. In consequence, a nonplane wave description of such beams is necessary. The most popular solutions of this problem are based on the paraxial approximation of the wave equation (small angle between the wave vector and the optical axis). Under this assumption various solutions (such as the Gauss beam, Hermite-Gaussian, Laguerre-Gaussian modes and others) were extensively studied. However, for pulses with a very short duration or ultraintense lasers (higher focusing increases the diffraction angle and intensity) the paraxial approximation can no longer be valid; see e.g., $[41,42]$ and references therein. Thus it would be desirable to go beyond the paraxial approximation. Various approaches to the solution of this problem were proposed. In Ref. [39] the authors construct auxiliary electromagnetic backgrounds which captures some essential features of transverse magnetic beams near the beam axis. Next, they show that the dynamics of a charged particle in such models can be explicitly discussed (without approximation); in this way they obtain some insight into predictions and analyses for experiments with intense or ultrashort lasers. More precisely, the following electromagnetic fields (see [39]):

$\vec{E}(x)=\mathcal{E}(u)\left(x^{1}, x^{2}, \sqrt{2} u\right), \quad \vec{B}(x)=\mathcal{E}(u)\left(-x^{2}, x^{1}, 0\right)$,

were considered, where the function $\mathcal{E}(u)$ is picked, at least, at $u=0$. Such fields capture some essential features of the transverse magnetic beams near the beam axis, such as the polarization structure, the local rise of the transverse fields, and the suppression of the longitudinal field as well as satisy $\vec{E} \cdot \vec{B}=0$ while, in contrast to (3.5), $\vec{E}^{2}-\vec{B}^{2}>0$ (see also [39]).

In what follows, first, we shall show that such fields can emerge from the gravitational metrics if we slightly modify the correspondence between gravitational, given by (2.1), and electromagnetic fields. As before we put $A=H$ in the one-form (3.1); however, this time we add a new term with an arbitrary function $\mathcal{F}$,

$$
\hat{\mathcal{A}}=-\mathbf{x} \cdot H(u) \mathbf{x} d u+\mathcal{F}(u) d v .
$$

Then one gets

$$
\begin{array}{r}
\vec{E}=\left(f_{1}, f_{2}, \dot{\mathcal{F}}\right), \quad \vec{B}=\left(-f_{2}, f_{1}, 0\right), \\
j^{\mu}=\sqrt{2}\left(\operatorname{tr}(H)+\frac{1}{2} \ddot{\mathcal{F}}\right)(1,0,0,1), \quad j^{\mu} j_{\mu}=0,
\end{array}
$$

where $f_{1}$ and $f_{2}$ are given by (3.3). Now, repeating the previous considerations [i.e., using the Lorentz equations (3.6) and next integrating] one finds the following relation:

$$
m \frac{d u}{d \tau}=-e \mathcal{F}(u)-D
$$

where $D$ is a constant of integration [see also (6.4)]. Then the Lorentz equations in terms of the $u$ coordinate read (cf. [56])

$$
\ddot{\mathbf{x}}(e \mathcal{F}+D)+e \dot{\mathcal{F}} \dot{\mathbf{x}}=2 e H \mathbf{x},
$$




$$
\ddot{v}(e \mathcal{F}+D)+2 e \dot{\mathcal{F}} \dot{v}=-2 e \mathbf{x} \cdot H \dot{\mathbf{x}} .
$$

Let us consider the diagonal profile $H_{11}=H_{22}$, $H_{12}=H_{21}=0$, and the function

$$
\mathcal{F}(u)=2 \int_{-\infty}^{u} \bar{u} H_{11}(\bar{u}) d \bar{u}
$$

(we assume here the vanishing of the gravitational profile at plus/minus null infinity in such a way that the function $\mathcal{F}$ is well defined). Consequently, one gets the electromagnetic field given by (5.1) with

$$
\mathcal{E}(u)=\sqrt{2} H_{11}(u) .
$$

Of course, the function $\mathcal{F}$ can be chosen up to a (irrelevant) constant, and our choice $\mathcal{F}(-\infty)=0$, due to the asymptotic vanishing of the gravitational profile and (5.5), gives

$$
D=-p_{v},
$$

where $p_{v}<0$ [cf. (3.8)]. Finally, let us note that the choice of the matrix $H, \operatorname{tr}(H) \neq 0$, corresponds to the, nonvacuum, generalized plane gravitational waves (here, in general, we do not assume that the weak energy condition holds) and, consequently, gives nonvacuum electromagnetic fields.

The general solution of Eqs. (5.6) was obtained in Ref. [39] by means of some integrals of motion. Here we apply a slightly different method. First, let us recall that having a particular solution to the second-order homogenous linear differential equation, the general solution can be expressed in terms of some integrals containing this solution. For a diagonal matrix $H$ and (5.8) the functions $x_{1}^{i}(u)=u$ are particular solutions to the transverse Lorentz force equation (5.6). Thus using the above property of ordinary differential equations we obtain that the general solution is a linear combination of $x_{1}^{i}$ and the second solution of the form

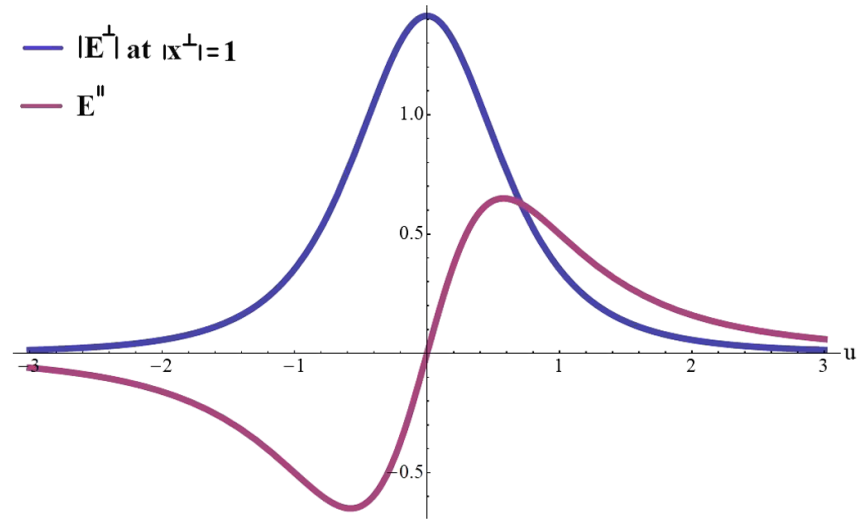

$$
x_{2}^{i}(u)=u \int \frac{d u}{u^{2}\left(e \mathcal{F}(u)-p_{v}\right)}
$$

[in the case of the longitudinal direction Eq. (5.7) can be integrated once [39] ].

Now, taking $H_{11}=H_{11}^{(1)}$ given by (2.6) one gets

$$
\mathcal{F}(u)=\frac{-a}{u^{2}+\epsilon^{2}},
$$

and the following electromagnetic vector field

$$
\begin{aligned}
\vec{E} & =\frac{\sqrt{2} a}{\left(u^{2}+\epsilon^{2}\right)^{2}}\left(x^{1}, x^{2}, \sqrt{2} u\right), \\
\vec{B} & =\frac{\sqrt{2} a}{\left(u^{2}+\epsilon^{2}\right)^{2}}\left(-x^{2}, x^{1}, 0\right),
\end{aligned}
$$

which was studied, in another gauge, in [39]. More precisely, it was shown there that the transverse Lorentz equations are explicitly solvable; moreover, there exist solutions exhibiting the focusing property. Namely, for the electromagnetic field (5.13) the conditions (2.11) lead to the following solutions to Eqs. (5.6):

$\mathbf{x}(u)=\frac{\mathbf{x}_{\text {in }}}{1-g}\left[1+\frac{u g}{\epsilon \sqrt{1-g}}\left(\frac{\pi}{2}+\tan ^{-1}\left(\frac{u}{\epsilon \sqrt{1-g}}\right)\right)\right]$,

where

$$
g \equiv \frac{e a}{-p_{v} \epsilon^{2}}
$$

satisfies $g<1$. Now, one can show that for $g<0$ there is a point $u_{0}$ such that $x^{i}\left(u_{0}\right)=0$, i.e., focusing; for $g>0$ there is no focusing. For example, taking $a=-\operatorname{sgn}(e)$ one gets the focusing case $g=\frac{|e|}{\epsilon^{2} p_{v}}<0$. The transverse and longitudinal electric field profiles of (5.13) as well as solutions (5.14) are plotted in Fig. 1.

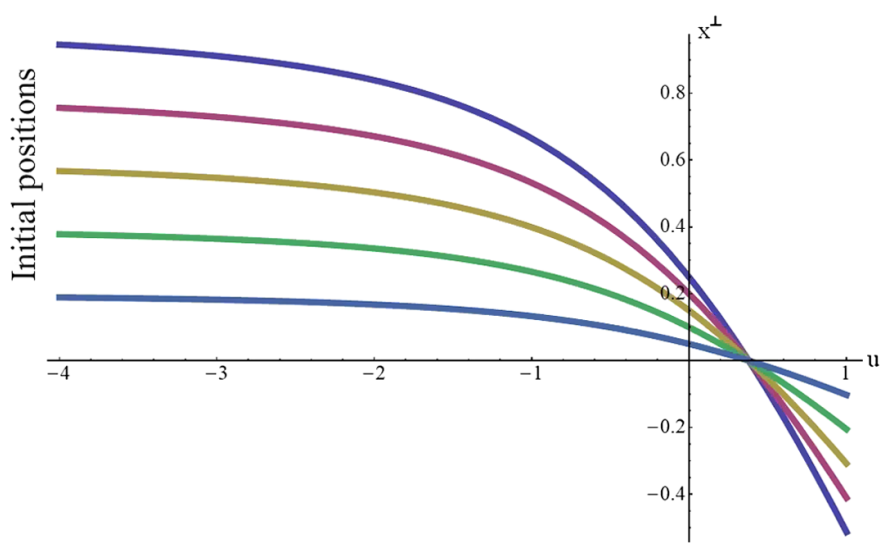

FIG. 1. The transverse and longitudinal electric field profiles corresponding to (5.13), i.e., (5.16) with $r=0$, as well as the transverse part of focusing solutions (5.14) $\left(e=-1, a=\epsilon=1, p_{v}=-1 / 3\right)$. 
For $g \geq 1$ the solutions are also expressible in terms of elementary functions (they consist of composition of logarithmic and rational functions); however, they exhibit singularities even though the starting potential is regular. This fact is related to the coefficient $e A_{v}-p_{v}$ appearing in front the second derivative in Eqs. (5.6) and (5.7). The condition $g<1$ ensures that this coefficient is never zero. Then for the continuous potential the solutions of these equations are regular. If there are some points where this coefficient vanishes (here $g \geq 1$ ), then the order of the differential equations is at most one and then singularities can appear. This is the reason for which starting from the continuous potential we arrive, for some physical parameters, at singular solutions. Moreover, the vanishing of this coefficient implies [see Eq. (5.5)] that reparametrization is valid only on some intervals (not globally defined) which makes considerations more complicated. In view of the above here and below we skipped such singular solutions as physically less transparent.
In Ref. [39] it was suggested that it would be interesting to analyze explicitly an example of the electric field (5.1) with multiple field oscillations [in contrast to (5.13)]. To this end we consider a counterpart of the circularly polarized plane gravitational waves (2.7). Namely, let us put $\gamma=r \epsilon, r>0$ in $H^{(2)}$ [see (2.7) and (2.8)] and consider the profile

$$
\begin{aligned}
& H_{11}=H_{22}=\frac{a}{\left(u^{2}+\epsilon^{2}\right)^{2}} \cos \left(2 r \tan ^{-1}(u / \epsilon)\right), \\
& H_{12}=H_{21}=0 .
\end{aligned}
$$

Then the function $\mathcal{F}$ is as follows: for $r=1$

$$
\mathcal{F}(u)=\frac{a u^{2}}{\left(u^{2}+\epsilon^{2}\right)^{2}}
$$

and for $r \neq 1$

$$
\mathcal{F}(u)=a \frac{2 \epsilon r u \sin \left(2 r \tan ^{-1}(u / \epsilon)\right)+\left(\epsilon^{2}-u^{2}\right) \cos \left(2 r \tan ^{-1}(u / \epsilon)\right)}{2 \epsilon^{2}\left(r^{2}-1\right)\left(u^{2}+\epsilon^{2}\right)}+\frac{a \cos (\pi r)}{2 \epsilon^{2}\left(r^{2}-1\right)} .
$$

Let us begin with the case of $r=1$. Then

$$
\mathcal{E}(u)=\sqrt{2} a \frac{\epsilon^{2}-u^{2}}{\left(u^{2}+\epsilon^{2}\right)^{3}},
$$

and the transverse part of the electromagnetic field is picked at $u=0$ and has two zeros (in contrast to the profile discussed in [39]). In what follows we shall show that the trajectories as well as the focusing conditions can also be explicitly written down.

To this end let us consider three cases distinguished by the value of the constant $g$ given by Eq. (5.15). First, let $g>0$. Imposing (2.11) one gets the solution

$$
\mathbf{x}(u)=\mathbf{x}_{\mathrm{in}}\left[1+\frac{g u}{\sqrt{4 g+g^{2}}}\left(\frac{1}{\sqrt{A_{-}}}\left(\frac{\pi}{2}+\tan ^{-1}\left(\frac{u}{\sqrt{A_{-}}}\right)\right)-\frac{1}{\sqrt{A_{+}}}\left(\frac{\pi}{2}+\tan ^{-1}\left(\frac{u}{\sqrt{A_{+}}}\right)\right)\right)\right],
$$

where

$$
A_{ \pm} \equiv \frac{\epsilon^{2}}{2}\left(2+g \pm \sqrt{4 g+g^{2}}\right)>0
$$

Using $g>0$ one can show, after some calculations, that there is no focusing.

Next, when $0>g>-4$, we have

$$
\mathbf{x}(u)=\mathbf{x}_{\mathrm{in}}\left[1+\frac{g u}{4 B}\left(\pi+\tan ^{-1}\left(\frac{u+\epsilon \sqrt{-g / 4}}{B}\right)+\tan ^{-1}\left(\frac{u-\epsilon \sqrt{-g / 4}}{B}\right)\right)-\frac{\sqrt{-g} u}{2 \epsilon} \tanh ^{-1}\left(\frac{\epsilon \sqrt{-g} u}{u^{2}+\epsilon^{2}}\right)\right],
$$

where

$$
B=\epsilon \sqrt{1+g / 4} .
$$




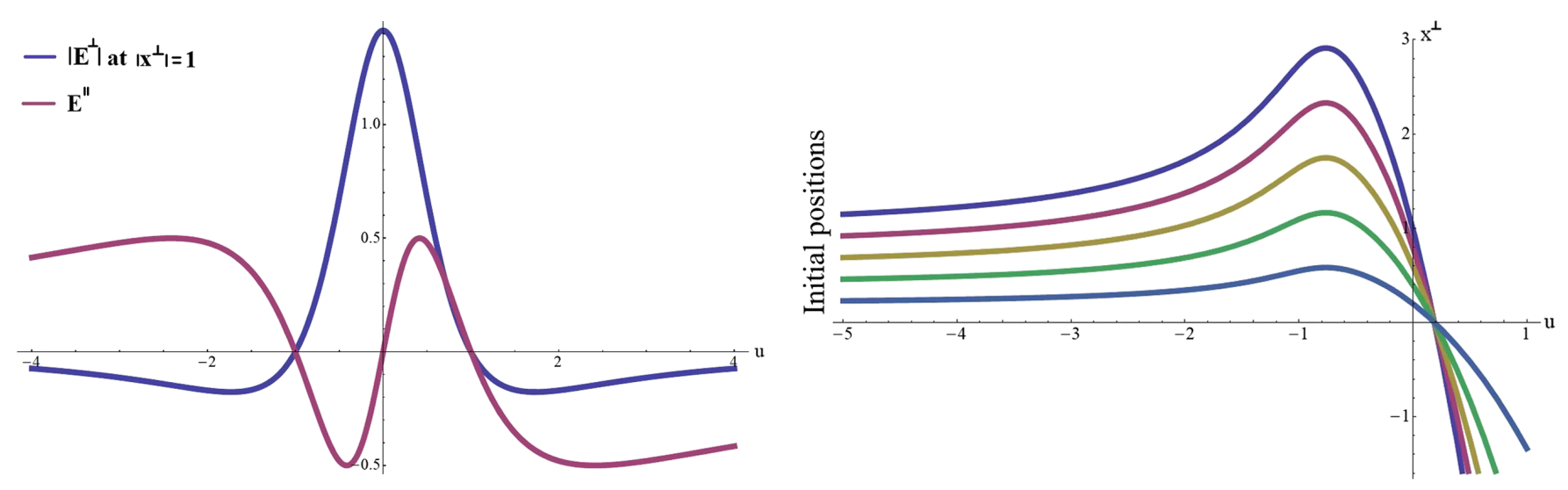

FIG. 2. The transverse and longitudinal electric field profiles corresponding to (5.19), i.e., (5.16) with $r=1$, as well as the transverse part of focusing solutions (5.22) $\left(e=-1, a=\epsilon=1, p_{v}=-1 / 3\right)$.

Then there is a point $u_{0}$ where $x^{i}\left(u_{0}\right)=0$ for all initial points, i.e., focusing. Let us note that taking $a=-\operatorname{sgn}(e)$ one gets $g=\frac{|e|}{p_{v} \epsilon^{2}}<0$; however, only for some values of the particle parameters does the condition $g>-4$ hold [in contrast to the electromagnetic field (5.13), nonvanishing in the transverse direction]. The transverse and longitudinal electric field profiles corresponding to (5.19) as well as the focusing solutions (5.22) are illustrated in Fig. 2.

Finally, for $g \leq-4$ the solutions can also be expressed in terms of elementary functions; however, they exhibit some singularities, so thus we skip them here [see the discussion after (5.15)].

For arbitrary function $\mathcal{F}$ the integral in (5.11) cannot be explicitly computed, even when the functions $\mathcal{E}$ and $\mathcal{F}$ are both rational ones [e.g., $\mathcal{F}$ given by (5.16) with the nonnegative integer $r$ ]. Thus, in what follows we give some sufficient conditions to ensure the focusing property of the electromagnetic field (5.1), and next we apply them to the case (5.16).

To begin with, we should assume that the denominator in the integral (5.11) does not vanish anywhere

$$
e \mathcal{F}(u)-p_{v} \neq 0
$$

this can be achieved by a suitable choice of the range of parameters $e$ and/or $p_{v}$ (since $\mathcal{F}$ tends to zero at the null infinities). Next, we rewrite the general solution in the following form:

$$
\begin{aligned}
x^{i}(u) & =C_{1}^{i} x_{1}^{i}(u)+C_{2}^{i} x_{2}^{i}(u) \\
& =C_{1}^{i} u+\frac{C_{2}^{i} p_{v}}{e \mathcal{F}(0)-p_{v}}+C_{2}^{i} u \int_{-\infty}^{u} \frac{\mathcal{G}(\bar{u}) d \bar{u}}{\bar{u}^{2}\left(e \mathcal{F}(\bar{u})-p_{v}\right)},
\end{aligned}
$$

where

$$
\mathcal{G}(u)=\frac{-e p_{v}(\mathcal{F}(0)-\mathcal{F}(u))}{e \mathcal{F}(0)-p_{v}}
$$

Then the function under the integral (5.25) is well defined on the whole real line since $\mathcal{G}(0)=0$ and, by virtue of (5.8), $\dot{\mathcal{G}}(0)=0$. Now imposing the conditions (2.11) one obtains $C_{1}^{i}=0$ and, by virtue of l'Hôpital's rule, the following form of the solution:

$$
\mathbf{x}(u)=\mathbf{x}_{\text {in }}\left(-u \int_{-\infty}^{u} \frac{\mathcal{G}(\bar{u}) d \bar{u}}{\bar{u}^{2}\left(e \mathcal{F}(\bar{u})-p_{v}\right)}-\frac{p_{v}}{e \mathcal{F}(0)-p_{v}}\right) .
$$

Hence, the inequality

$$
\frac{1}{\left(e \mathcal{F}(0)-p_{v}\right)} \int_{-\infty}^{\infty} \frac{e(\mathcal{F}(0)-\mathcal{F}(u)) d u}{u^{2}\left(e \mathcal{F}(u)-p_{v}\right)}>0,
$$

together with (5.24), implies a focusing point. For instance, the condition (5.28) is satisfied when the function $e \mathcal{F}(u)$ has the global maximum at $u=0$,

$$
e \mathcal{F}(0) \geq e \mathcal{F}(u)
$$

One can check that in the case of the electromagnetic field defined by (5.13) and (5.19) [equivalently, (5.16) with $r=0,1]$ the criteria (5.24) and (5.29) yield the conditions obtained above. Furthermore, applying these criteria to the electromagnetic field defined by (5.16) with $0<r<1$ we arrive at the following focusing conditions:

$$
\begin{gathered}
\text { for } 0<r \leq \frac{1}{2}, \quad g<0 \\
\text { for } \frac{1}{2}<r<1, \quad \frac{2\left(1-r^{2}\right)}{\cos (\pi r)+r \sin \left(\frac{\pi}{2 r}\right)}<g<0 .
\end{gathered}
$$

Taking into account the above discussed case $r=1$, we see explicitly that when the profile of the electromagnetic field 

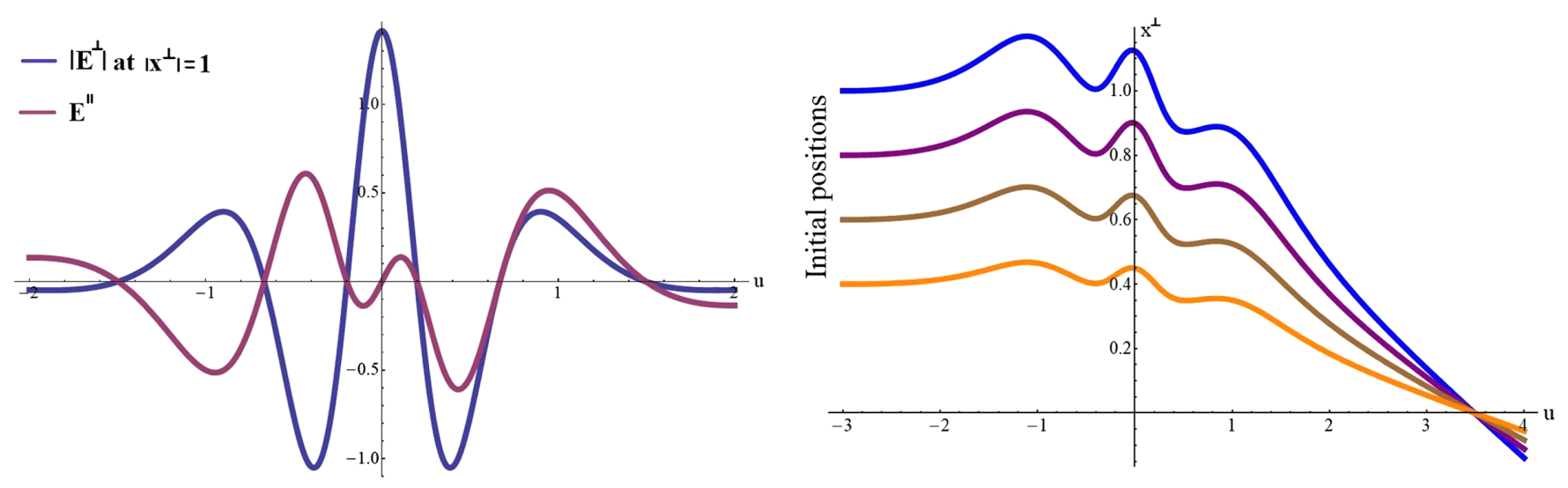

FIG. 3. The transverse and longitudinal electric field profiles corresponding to (5.16) with $r=4$ as well as the transverse part of focusing solutions $\left(e=-1, a=\epsilon=1, p_{v}=-1 / 2\right)$.

vanishes at some points [see (5.9) and (5.16) with $\frac{1}{2}<r \leq 1$ ], then there are some additional restrictions for the particle parameters [see (5.30)]. Finally, it is worthwhile to notice that (5.30) gives a new Kober's-type inequality [70].

Finally, let us remember that differentiating (5.27) and using (5.8) as well as (5.26) one obtains the relation

$$
\ddot{\mathbf{x}}=\frac{-p_{v} e \mathcal{E}(u) \mathbf{x}_{\mathrm{in}}}{\left(e \mathcal{F}-p_{v}\right)^{2}}
$$

thus the zeros of the electromagnetic profile $\mathcal{E}$ are strictly related to the inflection points of the solutions (5.27); see Figs. 1-3 (e.g., for $r=0$ and $g<0$, Fig. 1, they are concave).

\section{CONFORMAL SYMMETRY AND INTEGRALS OF MOTION}

To obtain a wider view on the results discussed above let us have a look at them from the integrals of motion point of view. The discussion of the integrability of the electromagnetic fields of the type which appear in previous sections were partially elaborated in Refs. [36,39,40]. In particular, it was shown there that such systems are superintegrable. However, let us stress that such (super) integrability does not ensure that the equations of motion are explicitly solvable. In view of this the question is whether there are new integrals of motion (or even bettersymmetry generators) which can help to find explicit solutions. Let us study this problem in more detail for the electromagnetic vortices discussed in Secs. III and IV. To obtain the more complete picture of this problem first, following Refs. [36,39,40] (however, applying our conventions of the signature and gauge fixing), we present the general discussion of the integrability.

Let us start with the Lagrangian of a relativistic particle in an electromagnetic background $A_{\mu}$,

$$
L=-m \sqrt{-\frac{d x^{\mu}}{d \tau} \frac{d x_{\mu}}{d \tau}}+e A_{\mu} \frac{d x^{\mu}}{d \tau} .
$$

Since $L$ is homogeneous of the first degree in velocities (it is invariant under reparametrization), the Hamiltonian (evolution generator) of the particle vanishes. To remedy this problem and to describe the dynamical evolution, one has to fix the gauge by choosing a time parameter $\tau=\tau\left(x^{\mu}\right)$ (of course, all choices lead to equivalent results; however, some of them can be more useful for given systems); the most popular is the affine parametrization. On the other hand, for the discussed systems the light-cone coordinates are more suitable. In these coordinates the Lagrangian and the affinity condition take the form

$$
\begin{gathered}
L=-m \sqrt{-2 \frac{d u}{d \tau} \frac{d v}{d \tau}-\left(\frac{d \mathbf{x}}{d \tau}\right)^{2}}+e A_{u} \frac{d u}{d \tau}+e A_{v} \frac{d v}{d \tau}+e \mathbf{A} \frac{d \mathbf{x}}{d \tau} \\
2 \frac{d u}{d \tau} \frac{d v}{d \tau}+\left(\frac{d \mathbf{x}}{d \tau}\right)^{2}=-1
\end{gathered}
$$

Now, let us assume that the potential $A_{\mu}$ of the electromagnetic field can be chosen as follows: $\mathbf{A}=\mathbf{A}(u, \mathbf{x})$, $A_{u}=A_{u}(u, \mathbf{x})$, and $A_{v}=A_{v}(u)$ (such a situation includes all the above considered cases). Then the form of the potential determines the function $\tau\left(x^{\mu}\right)$ uniquely. Indeed, the canonical momentum $p_{v}$ conjugated to the $v$ coordinate is an integral of motion. In consequence, the condition (6.3) yields

$$
p_{v}=m \frac{d u}{d \tau}+e A_{v}(u)=\text { const },
$$

which implies the form of the affine parameter $\tau=\tau(u)$. To make this reparametrization invertible one should assume that the derivative $\frac{d u}{d \tau}$ is of constant sign (positive or negative). Furthermore, for the free particle 
$p_{v}=p^{u}=\left(p^{3}-p^{0}\right) \sqrt{2}<0$, thus assuming that $A_{v}$ vanishes at null infinities, one obtains $\frac{d u}{d \tau}<0$. Then

$$
L=m \sqrt{-2 \dot{v}-\dot{\mathbf{x}}^{2}}+e A_{u}+e A_{v} \dot{v}+e \mathbf{A} \cdot \dot{\mathbf{x}} .
$$

Finally, the condition (6.3) takes the form

$$
\dot{\mathbf{x}}^{2}+\frac{m^{2}}{\left(p_{v}-e A_{v}\right)^{2}}=-2 \dot{v} .
$$

Of course, the above Lagrangian together with the condition (6.6) implies the suitable equations of motion.

Now, we are in the position to form the Hamiltonian formalism. The phase space obtained is six dimensional, $\left(v, p_{v}\right)$ and $(\mathbf{x}, \mathbf{p})$ with the canonical Poisson brackets, and the Hamiltonian is of the following form:

$$
H=\frac{(\mathbf{p}-e \mathbf{A})^{2}+m^{2}}{2\left(p_{v}-e A_{v}\right)}-e A_{u} .
$$

Moreover, let us note that combining Eqs. (6.4) and (6.6) one obtains the relation $p_{u}=-H$.

Let us now discuss the problem of integrals of motion. First, let us consider the electromagnetic potential corresponding to the plane gravitational waves (2.1), i.e., $A_{u}(u, \mathbf{x})=-\mathbf{x} \cdot A(u) \mathbf{x}, \mathbf{A}=0$, and $A_{v}=0$, where $A(u)$ is a symmetric matrix. Then the Hamiltonian (6.7) yields the desired equations of motion (3.9) and (3.10). As we indicated above $p_{v}$ is an integral of motion. However, it turns out that [36] the quantities

$$
I_{\mathbf{k}}=\mathbf{k} \cdot \mathbf{p}-p_{v} \mathbf{x} \cdot \dot{\mathbf{k}}
$$

where $\mathbf{k}=\mathbf{k}(u)$ is a solution of the following set of equations [cf. Eqs. (3.9)], and

$$
\ddot{\mathbf{k}}=\frac{2 e}{-p_{v}} A \mathbf{k}
$$

are also integrals of motion. Of course, $I_{\mathbf{k}}$ and $p_{v}$ are in involution. Moreover, by choosing four independent solutions of the set of Eqs. (6.9) and noting that the Wronskian of these solutions is independent of $u$ (in consequence it can be determined at one point), $I_{\mathbf{k}}$ yields four constants of motion forming two (independent) copies of the Heisenberg algebra. Summarizing, we have three integrals in involution and the two additional integrals; thus the motion in these electromagnetic fields is maximally superintegrable. However, let us stress that even such superintegrability does not ensure the explicit solutions of the equations of motion (3.9) and (3.10) and does not select any special profile $A$.

To better understand the explicit solvability of the discussed electromagnetic vortices (3.15) and (3.16), let us recall that with any Killing vector field $K$, e.g., a Poincaré generator in the Minkowski spacetime, one can associate an integral of motion of the free particle. Of course, when an electromagnetic background is switched on, then usually this integral is not a conserved charge. However, if the Lie derivative of the potential $\mathcal{A}=A_{\mu} d x^{\mu}$ satisfies the following condition:

$$
\mathcal{L}_{K} \mathcal{A}=d \chi,
$$

with a function $\chi$ (i.e., $\mathcal{L}_{K} F=0$ ), then

$$
I_{K}=K_{\mu} P^{\mu}-e \chi=K_{\mu}\left(m \frac{d x^{\mu}}{d \tau}+e A_{\mu}\right)-e \chi,
$$

is an integral of motion.

Now, let us assume that $K$ is a conformal vector field with the conformal factor $2 \psi$ such that (6.10) holds. Then along the trajectories one obtains

$$
\frac{d I_{K}}{d \tau}=m \psi
$$

The key observation is that if $\psi$ is a function of $\tau$, then it can be rewritten as a derivative of some function of $\tau$; in consequence one obtains a new, $\tau$ dependent, integral of motion [for example, if $K$ is a homothetic vector field satisfying (6.10), then $\psi=\psi_{0}$ is a constant and consequently $I_{K}-m \tau \psi_{0}$ is an integral of motion].

Let us apply the above approach to the electromagnetic vortices discussed in Secs. III and IV. To this end let us recall that the space of conformal vector fields on the Minkowski spacetime is generated by the Killing fields together with three types (nonisometric) of conformal fields: radial, special, and general ones (see [20]). In particular, there is a standard special conformal vector $S$ (i.e., the one for which the gradient of the conformal factor is a parallel null vector along the $v$ coordinate); it is of the following form:

$$
S=u^{2} \partial_{u}-\frac{1}{2} \mathbf{x}^{2} \partial_{v}+u \mathbf{x} \cdot \nabla,
$$

and its conformal factor reads $\psi=u$. Since our aim is to find conformal vectors satisfying conditions (6.10) for the potential with $A^{(1)}$ [corresponding to (2.6)] we add to $S$ some Killing vectors (here Poincaré generators). Namely, by straightforward computations one checks that the following conformal vector field (obtained by adding the $u$-translation generator)

$K^{(1)}=S+\epsilon^{2} \partial_{u}=\left(u^{2}+\epsilon^{2}\right) \partial_{u}-\frac{1}{2} \mathbf{x}^{2} \partial_{v}+u \mathbf{x} \cdot \nabla$

satisfies

$$
\mathcal{L}_{K^{(1)}} \mathcal{A}^{(1)}=0,
$$


i.e., Eq. (6.10) with $\chi=0$. This fact perfectly agrees with the gravitational picture where $K^{(1)}$ is a conformal generator for the gravitational plane wave with the profile $H^{(1)}$ (see [21]). In consequence Eqs. (6.6) and (6.12) lead to the following integral of motion:

$$
\begin{aligned}
I^{(1)}= & -\frac{m^{2} \epsilon^{2}}{2 p_{v}^{2}}-e a \frac{\left(x^{1}\right)^{2}-\left(x^{2}\right)^{2}}{p_{v}\left(u^{2}+\epsilon^{2}\right)} \\
& -\left(u^{2}+\epsilon^{2}\right) \frac{\dot{\mathbf{x}}^{2}}{2}-\frac{1}{2} \mathbf{x}^{2}+u \mathbf{x} \cdot \dot{\mathbf{x}},
\end{aligned}
$$

which is of the same form as the one obtained for the plane gravitational wave from $K^{(1)}$; see [21] (more precisely, they coincide after replacement $a \rightarrow \frac{2 a e}{-p_{v}}$ in the gravitational case). In summary, we see that the conformal generator $K^{(1)}$ implies, in the case of the discussed electromagnetic vortices in the Minkowski spacetime, the same integral of motion (modulo coupling constants) as $K^{(1)}$ for the gravitational wave (2.1) with $H^{(1)}$ (but without the electromagnetic potential); the term arising from the electromagnetic potential coincides with the additional term appearing in $\dot{v}$ in the case of gravitational waves. This immediately allows us to understand better the role of the conformal symmetry in the explicit solvability of the equations of motion for the electromagnetic vortices (3.15). Indeed, following Ref. [21] $I^{(1)}$ can be rewritten as the sum of two independent Ermakov-Lewis invariants [43-45], namely

$$
\begin{aligned}
I^{(1)}= & -\frac{m^{2} \epsilon^{2}}{2 p_{v}^{2}}-\frac{\epsilon}{2}\left[\left(\rho \dot{x}^{1}-\dot{\rho} x^{1}\right)^{2}+\frac{\Lambda_{1}\left(x^{1}\right)^{2}}{\rho^{2}}\right] \\
& -\frac{\epsilon}{2}\left[\left(\rho \dot{x}^{2}-\dot{\rho} x^{2}\right)^{2}+\frac{\Lambda_{2}\left(x^{2}\right)^{2}}{\rho^{2}}\right]
\end{aligned}
$$

where $\Lambda_{i}$ are defined by Eqs. (2.10) with replacement $a \rightarrow \frac{2 a e}{-p_{v}}$ and $\rho$ is of the form

$$
\rho(u)=\frac{\sqrt{u^{2}+\epsilon^{2}}}{\sqrt{\epsilon}} .
$$

Moreover, the function $\rho$ satisfies the set of the ErmakovMilne-Pinney equations for the profiles $A^{(1)}$ and $\tilde{A}^{(1)}$ (the latter one is a diagonal matrix with the constant elements $\left.-\Lambda_{i}, i=1,2\right)$,

$$
\ddot{\rho} I-A^{(1)} \rho=-\frac{\tilde{A}^{(1)}}{\rho^{3}} .
$$

This information can be used to find the solutions of the transverse part of the equations of motion. According to the general procedure (see e.g., [67]), the transformation

$$
\frac{d \tilde{u}}{d u}=\frac{1}{\rho^{2}(u)}, \quad \mathbf{x}=\sqrt{\epsilon} \rho(u) \tilde{\mathbf{x}},
$$

should relate the $u$-dependent linear oscillator, defined by $A^{(1)}$, to the harmonic one with the frequencies $\Lambda_{1,2}$. In our case, i.e., $\rho$ given by (6.18), the above formulas yield the Niederer transformation [Eqs. (4.1)] and consequently the explicit integrability. Furthermore, it turns out (see [67] and references therein) that the Ermakov-Lewis invariants can be interpreted as the "classical" energy in the new coordinates $\tilde{\mathbf{x}}, \tilde{u}$. In our case this leads to the identity

$$
I^{(1)}=-\epsilon^{2}\left[\frac{m^{2}}{2 p_{v}^{2}}+E^{(1)}\right],
$$

where

$E^{(1)}=\frac{1}{2} \tilde{\mathbf{x}}^{\prime 2}-\frac{1}{2} \tilde{\mathbf{x}} \cdot \tilde{H}^{(1)} \tilde{\mathbf{x}}=\frac{1}{2} \tilde{\mathbf{x}}^{\prime 2}+\frac{\Lambda_{1}\left(\tilde{x}^{1}\right)^{2}}{2}+\frac{\Lambda_{2}\left(\tilde{x}^{2}\right)^{2}}{2}$

and $\Lambda_{i}$ are defined by (2.10) with the above-mentioned replacement. In consequence, we obtain an interpretation of the integral of motion associated with the proper conformal generator $K^{(1)}$.

In the case of the potential $A^{(2)}$ defined by the profile (2.7) we have to add to $K^{(1)}$ (and consequently to $S$ ) another Poincaré generator. Namely, direct computations show that the conformal vector field

$$
K^{(2)}=K^{(1)}-\gamma\left(x^{2} \partial_{1}-x^{1} \partial_{2}\right)
$$

satisfies

$$
\mathcal{L}_{K^{(2)}} \mathcal{A}^{(2)}=0,
$$

i.e., Eq. (6.10) with $\chi=0$. Moreover, the conformal factor is the same as for $K^{(1)}$, i.e., $\psi=u$. In consequence, by virtue of Eqs. (6.6) and (6.12) one obtains the following integral of motion:

$$
\begin{aligned}
I^{(2)}= & -\frac{m^{2} \epsilon^{2}}{2 p_{v}^{2}}-\frac{e}{p_{v}}\left(u^{2}+\epsilon^{2}\right) \mathbf{x} \cdot A^{(2)} \mathbf{x} \\
& -\left(u^{2}+\epsilon^{2}\right) \frac{\dot{\mathbf{x}}^{2}}{2}-\frac{1}{2} \mathbf{x}^{2}+u \dot{\mathbf{x}} \cdot \mathbf{x}-\gamma \dot{\mathbf{x}} \times \mathbf{x},
\end{aligned}
$$

which again differs from the one obtained for the plane gravitational wave (2.1) with the profile (2.7) by the replacement $a \rightarrow \frac{2 a e}{-p_{v}}$ (cf. results in [21]). Furthermore, the function $\rho$ and profile $A^{(2)}$ satisfy the Ermakov-MilnePinney type equation [similar to Eq. (6.19)]. In consequence, they lead to the transformation (2.12); i.e., it allows one to find the explicit solution of the transversal equation (3.9). Finally, following [21] the integral of motion $I^{(2)}$ 
can be interpreted as the sum total energy for the system defined by the equations of motion (2.14), i.e.,

$$
I^{(2)}=-\epsilon^{2}\left[\frac{m^{2}}{2 p_{v}^{2}}+E^{(2)}\right]
$$

where

$$
E^{(2)}=\frac{1}{2}\left(\mathbf{y}^{\prime}\right)^{2}+\frac{1}{2} \Omega_{+}\left(y^{1}\right)^{2}+\frac{1}{2} \Omega_{-}\left(y^{2}\right)^{2}
$$

and $\Omega_{ \pm}$are given by Eqs. (2.15) with $a \rightarrow \frac{2 a e}{-p_{v}}$.

Summarizing, we have shown that some special conformal transformations of the Minkowski spacetime can be chosen in such a way that they preserve electromagnetic fields and consequently generate new integrals of motion. In the presented cases these integrals can be interpreted in terms of the Ermakov-Lewis invariants (Ermakov-MilnePinney equations) leading to the Niederer transformation and, consequently, explicit solutions in terms of new variables. Moreover these integrals can be interpreted as the total energy of the dynamical system in new coordinates. All these observations coincide with the gravitational picture where these conformal vector fields survive for the plane gravitational waves ([21] and references therein) obtained by means of the Kerr-Schild ansatz; the difference is that instead of the electromagnetic potential there is a suitable term in the gravitational metrics.

Finally, since the Kerr-Schild ansatz can be extended to the fixed backgrounds (see Sec. III) this suggests that the above observations concerning the conformal vector fields $K^{(1,2)}$ in the Minkowski spacetime should be extended to the electromagnetic potential $A^{(1,2)}$ in the fixed $g^{(1,2)}$ backgrounds. This fact can be checked directly by noting that the condition (6.10) is metric independent. This coincides with the results obtained in Sec. IV.

Above we discussed the integrability problem for the electromagnetic field defined by the potential (3.1). Finally, let us review these issues for the electromagnetic field considered in Sec. V, i.e., defined by

$$
\begin{aligned}
A_{u}(u, \mathbf{x}) & =-H_{11}(u) \mathbf{x}^{2}, \quad \mathbf{A}=0, \\
A_{v}(u) & =2 \int_{-\infty}^{u} \bar{u} H_{11}(\bar{u}) d \bar{u},
\end{aligned}
$$

where $H_{11}$ is an arbitrary function such that the last integral exists. Then the Hamiltonian formalism is given by (6.7) together with the above mentioned Poisson brackets [leading directly to Eqs. (5.6) and (5.7)]. Also in this case the canonical momentum $p_{v}$ is a constant of motion; the problem of other integrals of motion of such a system was discussed (in a slightly different gauge) in Refs. [39,40]. Namely, it was shown that each of the two following Poincaré generators $K^{(i)}$,

$$
K^{(i)}=u \partial_{i}-x^{i} \partial_{v}, \quad i=1,2,
$$

satisfy the condition (6.10) with the functions $\chi^{(i)}$ of the form

$$
\chi^{(i)}=-A_{v}(u) x^{i}, \quad i=1,2 .
$$

In consequence, the Killing vectors (6.29) generate two, $u$-dependent, integrals of motions

$$
I^{(i)}=-x^{i} p_{v}+u p_{i}+e x^{i} A_{v}(u), \quad i=1,2 .
$$

These integrals together with the previous one, $p_{v}$, are in involution, and thus the system governed by the potential (6.28) is integrable. Furthermore, it was shown that there is an additional integral of motion (which is not a polynomial in $p_{v}$ ) that makes this system (minimally) superintegrable. However, as in the case of the vortices discussed above this superintegrability does not ensure the explicit form of solutions. So the question is whether one can find other symmetry generators which distinguish the fields discussed in Sec. V and to give more transparent explanations of the explicit solvability or, in general, a more detailed discussion of the role of integrals of motion associated with the conformal symmetry (see the recent Refs. [71,72]). These issues are left for further investigations.

\section{CONCLUSIONS AND OUTLOOK}

Let us summarize. In the present work, using the idea of the classical double copy, we construct null electromagnetic fields which are explicitly solvable and directly generalize the electromagnetic vortices considered, in the context of singular optics, in Ref. [33] (in contrast to the latter ones, they form some pulses and the Dirac delta limit of the profile can easily be done). Since these results are strictly related to the notion of Niederer's transformation, we analyze the geometric extension of the latter in the case of the plane gravitational spacetimes endowed with the crossed electromagnetic fields (3.2) and (3.3); in this approach the transverse part of the Lorentz force equations transformed by the Niederer map can be obtained by means of a new metric (conformally related to the initial one) and a new, crossed, electromagnetic field. In consequence, for some special cases related to the conformal symmetry of spacetimes, the transverse Lorentz equations on a curved spacetime can also be analytically solvable. We also showed that these results possess their origin in additional integrals of motion associated with conformal generators preserving the discussed vortices and plane gravitational waves.

In the second part we showed that the electromagnetic backgrounds which capture some essential features of the transverse magnetic laser beam near the beam axis, proposed in Ref. [39], can also emerge from some gravitational counterparts. Moreover, one of the latter ones leads to the electromagnetic profile with zeros and is 
analytically solvable in the transverse directions; such a situation allowed us to discuss explicitly the focusing conditions. Since the focusing properties of electromagnetic fields seem to be especially interesting, thus we gave some criteria and apply them to the electromagnetic counterparts of some gravitational metrics. Finally, let us note that the form of the electromagnetic fields discussed does not suggest the solvability of the corresponding Lorentz force equations; however, in view of the role of conformal symmetry in gravity and the double copy conjecture, the integrability of dynamics governed by these special electromagnetic fields arises naturally (the existence of explicit solutions to the geodesic equations coincides with the existence of the explicit solutions to the Lorentz force equations). Such a situation suggests deeper relations between both gravitational and gauge theories in the double copy approach.

The results obtained can be extended in various directions. As usual the exact solvability at the classical level should have its reflection at the quantum level. Thus it would be interesting to consider the quantum picture of the results obtained here, including both gravitational and electromagnetic backgrounds (see e.g., [39,73]). This is especially interesting in view of the double copy and recent results presented in Ref. [74] for gravitational sandwiches.
Furthermore, let us recall that the Penrose limit of spacetimes yields the plane gravitational waves; thus, the question is which ones correspond to the distinguished plane wave spacetimes $g^{(1,2)}$ (cf. [9,48,49]). Moreover, the analytical solutions obtained can also be useful in the recent studies concerning some aspects of optical effects in the nonlinear plane gravitational waves $[75,76]$ as well as trapping problems in gravity [36-38]. Finally, following Refs. [41,42] and [77,78], we hope that they can also be useful in the study of the light-matter interaction, especially for strong focusing of short or intense laser pulses.

\section{ACKNOWLEDGMENTS}

The authors thank Piotr Kosiński for helpful discussions as well as Peter Horvathy for useful remarks and suggestions. Comments of Cezary Gonera, Joanna Gonera, and Paweł Maślanka are also acknowledged. We are also grateful to the anonymous referees for a number of useful suggestions which, we feel, improved substantially the readability of the paper as well as inspired some new results. This work has been partially supported by Grant No. 2016/23/B/ST2/00727 of National Science Centre, Poland.
[1] B. Abbott et al. (LIGO Scientific and Virgo Collaborations), GW170814: A Three-Detector Observation of Gravitational Waves from a Binary Black Hole Coalescence, Phys. Rev. Lett. 119, 141101 (2017).

[2] B. Abbott et al. (LIGO Scientific and Virgo Collaborations), GW170817: Observation of Gravitational Waves from a Binary Neutron Star Inspiral, Phys. Rev. Lett. 119, 161101 (2017).

[3] M. Favata, The gravitational-wave memory effect, Classical Quantum Gravity 27, 084036 (2010).

[4] P. Lasky, E. Thrane, Y. Levin, J. Blackman, and Y. Chen, Detecting Gravitational-Wave Memory with LIGO: Implications of GW150914, Phys. Rev. Lett. 117, 061102 (2016).

[5] P.-M. Zhang, C. Duval, G. Gibbons, and P. Horvathy, The memory effect for plane gravitational waves, Phys. Lett. B 772, 743 (2017).

[6] P.-M. Zhang, C. Duval, G. Gibbons, and P. Horvathy, Soft gravitons and the memory effect for plane gravitational waves, Phys. Rev. D 96, 064013 (2017).

[7] A. Strominger, On BMS invariance of gravitational scattering, J. High Energy Phys. 07 (2014) 152.

[8] A. Strominger and A. Zhiboedov, Gravitational memory, BMS supertranslations and soft theorems, J. High Energy Phys. 01 (2016) 086.

[9] G. Shore, Memory, penrose limits and the geometry of gravitational shockwaves and gyratons, J. High Energy Phys. 12 (2018) 133.
[10] T. Wang, J. Fier, B. Li, G. Lv, Z. Wang, Y. Wu, and A. Wang, Singularities of plane gravitational waves and their memory effects, arXiv:1807.09397.

[11] C. Moore, D. Mihaylov, A. Lasenby, and G. Gilmore, Astrometric Search Method for Individually Resolvable Gravitational Wave Sources with Gaia, Phys. Rev. Lett. 119, 261102 (2017).

[12] R. Penrose, The geometry of impulsive gravitational waves, in General Relativity, Papers in Honour of J. L. Synge, edited by L. O'Raifeartaigh (Clarendon Press, Oxford, 1972), p. 101.

[13] R. Steinbauer, Geodesics and geodesic deviation for impulsive gravitational waves, J. Math. Phys. (N.Y.) 39, 2201 (1998).

[14] J. Podolsky and K. Vesely, New examples of sandwich gravitational waves and their impulsive limit, Czech. J. Phys. 48, 871 (1998).

[15] M. Kunzinger and R. Steinbauer, A rigorous solution concept for geodesic and geodesic deviation equations in impulsive gravitational waves, J. Math. Phys. (N.Y.) 40, 1479 (1999).

[16] P.-M. Zhang, C. Duval, G. Gibbons, and P. Horvathy, Velocity memory effect for polarized gravitational waves, J. Cosmol. Astropart. Phys. 05 (2018) 030.

[17] K. Andrzejewski and S. Prencel, Memory effect, conformal symmetry and gravitational plane waves, Phys. Lett. B 782, 421 (2018). 
[18] B. Tupper, A. Keane, G. Hall, A. Coley, and J. Carot, Conformal symmetry inheritance in null fluid spacetimes, Classical Quantum Gravity 20, 801 (2003).

[19] A. Keane and B. Tupper, Conformal symmetry classes for pp-wave spacetimes, Classical Quantum Gravity 21, 2037 (2004).

[20] W. Kühnel and H.-B. Rademacher, Conformal geometry of gravitational plane waves, Geometriae Dedicata 109, 175 (2004).

[21] K. Andrzejewski and S. Prencel, Niederer's transformation, time-dependent oscillators and polarized gravitational waves, Classical Quantum Gravity 36, 155008 (2019).

[22] U. Niederer, The maximal kinematical invariance group of the harmonic oscillator, Helv. Phys. Acta 46, 191 (1973).

[23] G. Burdet, C. Duval, and M. Perrin, Time-dependent quantum systems and chronoprojective geometry, Lett. Math. Phys. 10, 255 (1985).

[24] R. Monteiro, D. O'Connell, and C. White, Black holes and the double copy, J. High Energy Phys. 12 (2014) 056.

[25] A. Luna, R. Monteiro, D. O'Connell, and C. White, The classical double copy for Taub-NUT spacetime, Phys. Lett. B 750, 272 (2015).

[26] N. Bahjat-Abbas, A. Luna, and C. White, The Kerr-Schild double copy in curved spacetime, J. High Energy Phys. 12 (2017) 004.

[27] A. Luna, R. Monteiro, I. Nicholson, A. Ochirov, D. O'Connell, N. Westerberg, and C. White, Perturbative spacetimes from Yang-Mills theory, J. High Energy Phys. 04 (2017) 069.

[28] W. Goldberger and A. Ridgway, Radiation and the classical double copy for color charges, Phys. Rev. D 95, 125010 (2017).

[29] C. Cheung, TASI lectures on scattering amplitudes, arXiv: 1708.03872.

[30] C. White, The double copy: Gravity from gluons, Contemp.Phys. 59, 109 (2018).

[31] A. Luna, R. Monteiro, I. Nicholson, and D. O'Connell, Type D spacetimes and the Weyl double copy, Classical Quantum Gravity 36, 065003 (2019).

[32] M. Gurses and B. Tekin, Classical double copy: KerrSchild-Kundt metrics from Yang-Mills theory, Phys. Rev. D 98, 126017 (2018).

[33] I. Białynicki-Birula, Particle Beams Guided by Electromagnetic Vortices: New Solutions of the Lorentz, Schrödinger, Klein-Gordon and Dirac Equations, Phys. Rev. Lett. 93, 020402 (2004).

[34] I. Białynicki-Birula, Z. Białynicka-Birula, and N. Drozd, Trapping of charged particles by Bessel beams, in The Angular Momentum of Light (Cambridge University Press, Cambridge, England, 2012), p. 264.

[35] G. Gbur, Singular Optics (CRC Press, Boca Raton, FL, 2017).

[36] A. Ilderton, Screw-symmetric gravitational waves: A double copy of the vortex, Phys. Lett. B 782, 22 (2018).

[37] P.-M. Zhang, M. Cariglia, C. Duval, M. Elbistan, G. Gibbons, and P. Horvathy, Ion traps and the memory effect for periodic gravitational waves, Phys. Rev. D 98, 044037 (2018).

[38] I. Białynicki-Birula and S. Charzyński, Trapping and Guiding Bodies by Gravitational Waves Endowed with Angular Momentum, Phys. Rev. Lett. 121, 171101 (2018).
[39] T. Heinzl and A. Ilderton, Exact Classical and Quantum Dynamics in Background Electromagnetic Fields, Phys. Rev. Lett. 118, 113202 (2017).

[40] T. Heinzl and A. Ilderton, Superintegrable relativistic systems in spacetime-dependent background fields, J. Phys. A 50, 345204 (2017).

[41] W. Waters and B. King, On beam models and their paraxial approximation, Laser Phys. 28, 015003 (2018).

[42] C. Harvey, In Situ characterization of ultraintense laser pulses, Phys. Rev. Accel. Beams 21, 114001 (2018).

[43] H. Lewis, Classical and Quantum Systems with TimeDependent Harmonic-Oscillator-Type Hamiltonians, Phys. Rev. Lett. 18, 510 (1967).

[44] H. Lewis, Class of exact invariants for classical and quantum time-dependent harmonic oscillators, J. Math. Phys. (N.Y.) 9, 1976 (1968).

[45] H. Lewis and W. Riesenfeld, Class of Exact invariants for classical and quantum time-dependent harmonic oscillators, J. Math. Phys. (N.Y.) 10, 1458 (1969).

[46] V. Ermakov, Second order differential equations. Conditions of complete integrability, Univ. Izv. Kiev, Series III 9, 1 (1880); [A. Harin, under redaction by P. Leach, Appl. Anal. Discrete Math. 2, 123 (2008)].

[47] O. Kirillov and M. Levi, Rotating saddle trap as Foucault's pendulum, Am. J. Phys. 84, 26 (2016).

[48] M. Blau, M. Borunda, M. O'Loughlin, and G. Papadopoulos, Penrose limits and spacetime singularities, Classical Quantum Gravity 21, L43 (2004).

[49] M. Blau, M. Borunda, M. O'Loughlin, and G. Papadopoulos The Universality of penrose limits near space-time singularities, J. High Energy Phys. 07 (2004) 068.

[50] G. Hall and J. Steele, Conformal vector fields in general relativity, J. Math. Phys. (N.Y.) 32, 1847 (1991).

[51] J. Ehlers and W. Kundt, Exact solutions of the gravitational field equations, in Gravitation: An Introduction to Current Research, edited by L. Witten (Wiley, New York, 1962), p. 49.

[52] D. Eardley, J. Isenberg, J. Marsden, and V. Moncrief, Homothetic and conformal symmetries of solutions to Einstein's equations, Commun. Math. Phys. 106, 137 (1986).

[53] R. Maartens and S. Maharaj, Conformal symmetries of pp-waves, Classical Quantum Gravity 8, 503 (1991).

[54] P.-M. Zhang, G. Gibbons, and P. Horvathy, Kohn's theorem and Newton-Hooke symmetry for Hill's equations, Phys. Rev. D 85, 045031 (2012).

[55] P.-M. Zhang, P. Horvathy, K. Andrzejewski, J. Gonera, and P. Kosiński, Newton-Hooke type symmetry of anisotropic oscillators, Ann. Phys. (Amsterdam) 333, 335 (2013).

[56] V. Bagrov, M. Baldiotti, and D. Gitman, Charged particles in crossed and longitudinal electromagnetic fields and beam guides, J. Math. Phys. (N.Y.) 48, 082305 (2007).

[57] V. Bagrov and D. Gitman, Exact Solutions of Relativistic Wave Equations (Kluwer, Dordrecht, the Netherlands, 1990).

[58] W. Bonnor, Solutions of Maxwell's equations for charge moving with the speed of light, Int. J. Theor. Phys. 2, 373 (1969).

[59] I. Białynicki-Birula and Z. Białynicka-Birula, Vortex lines of the electromagnetic field, Phys. Rev. A 67, 062114 (2003).

[60] I. Białynicki-Birula, Electromagnetic vortex lines riding atop null solutions of the Maxwell equations, J. Opt. A 6 , S181 (2004). 
[61] I. Białynicki-Birula, Z. Białynicka-Birula, and B. Chmura, Trojan states of electrons guided by Bessel beams, Laser Phys. 15, 1371 (2005).

[62] S. Takagi, Equivalence of a harmonic oscillator to a free particle, Prog. Theor. Phys. 84, 1019 (1990).

[63] R. Carles, Linear vs. nonlinear effects for nonlinear Schrödinger equations with potential, Commun. Contemp. Math. 07, 483 (2005).

[64] K. Andrzejewski, J. Gonera, and P. Kosiński, Free-particle wave function and Niederer's transformation, Phys. Rev. A 89, 014103 (2014).

[65] C. Duval, G. Burdet, H. Kunzle, and M. Perrin, Bargmann structures and Newton-Cartan theory, Phys. Rev. D 31, 1841 (1985).

[66] M. Cariglia, C. Duval, G. Gibbons, and P. Horvathy, Eisenhart lifts and symmetries of time-dependent systems, Ann. Phys. (Amsterdam) 373, 631 (2016).

[67] M. Cariglia, A. Galajinsky, G. Gibbons, and P. Horvathy, Cosmological aspects of the Eisenhart-Duval lift, Eur. Phys. J. C 78, 314 (2018).

[68] C. Duval, G. Gibbons, and P. Horvathy, Celestial mechanics, conformal structures and gravitational waves, Phys. Rev. D 43, 3907 (1991).

[69] C. Duval, G. Gibbons, P. Horvathy, and P.-M. Zhang, Carroll symmetry of plane gravitational waves, Classical Quantum Gravity 34, 175003 (2017).
[70] D. Mitrinović, Analytic Inequalities (Springer-Verlag, Berlin, 1970).

[71] N. Dimakis, P. Terzis, and T. Christodoulakis, Integrability of geodesic motions in curved manifolds through non-local conserved charges, Phys. Rev. D 99, 104061 (2019).

[72] P.-M. Zhang, M. Cariglia, M. Elbistan, and P. Horvathy, Scaling and conformal symmetries for plane gravitational waves, arXiv:1905.08661.

[73] J. Garriga and E. Verdaguer, Scattering of quantum particles by gravitational plane waves, Phys. Rev. D 43, 391 (1991).

[74] T. Adamo, E. Casali, L. Mason, and S. Nekovar, Scattering on plane waves and the double copy, Classical Quantum Gravity 35, 015004 (2018).

[75] A. Harte, Strong lensing, plane gravitational waves and transient flashes, Classical Quantum Gravity 30, 075011 (2013).

[76] D. Bini, P. Fortini, A. Geralico, M. Haney, and A. Ortolan, Light scattering by radiation fields: The optical medium analogy, Europhys. Lett. 102, 20006 (2013).

[77] I. Białynicki-Birula and T. Radozycki, Pinning and transport of cyclotron/Landau orbits by electromagnetic vortices, Phys. Rev. A 73, 052114 (2006).

[78] T. Heinzl and A. Ilderton, A Lorentz and gauge invariant measure of laser intensity, Opt. Commun. 282, 1879 (2009). 